\title{
Activation of Locomotion in Adult Chronic Spinal Rats Is Achieved by Transplantation of Embryonic Raphe Cells Reinnervating a Precise Lumbar Level
}

\author{
Minerva Giménez y Ribotta, ${ }^{1}$ Jeanine Provencher,, ${ }^{2}$ Delphine Feraboli-Lohnherr, ${ }^{3}$ Serge Rossignol, ${ }^{2}$ \\ Alain Privat, ${ }^{1}$ and Didier Orsal ${ }^{3}$ \\ 1/nstitut National de la Santé et de la Recherche Médicale U336, Ecole Pratique des Hautes Etudes, Université \\ Montpellier II, F-34095 Montpellier, France ²Centre de Recherches en Sciences Neurologiques, Université de Montréal, \\ Montréal, Quebec, H3C 3J7 Canada and ${ }^{3}$ Centre National de la Recherche Scientifique EP 1848, Neurophysique et \\ Physiologie des Systèmes Moteurs, Université Rene Descartes, 75270 Paris, France
}

Traumatic lesions of the spinal cord yield a loss of supraspinal control of voluntary locomotor activity, although the spinal cord contains the necessary circuitry to generate the basic locomotor pattern. In spinal rats, this network, known as central pattern generator (CPG), was shown to be sensitive to serotonergic pharmacological stimulation. In previous works we have shown that embryonic raphe cells transplanted into the sublesional cord of adult rats can reinnervate specific targets, restore the lesion-induced increase in receptor densities of neurotransmitters, promote hindlimb weight support, and trigger a locomotor activity on a treadmill without any other pharmacological treatment or training.

With the aim of discriminating whether the action of serotonin on CPG is associated to a specific level of the cord, we have transplanted embryonic raphe cells at two different levels of the sublesional cord (T9 and T11) and then performed analysis of the kinematic and EMG activity synchronously recorded during locomotion. Locomotor performances were correlated to the reinnervated level of the cord and compared to that of intact and transected nontransplanted animals. The movements expressed by T11 transplanted animals correspond to a well defined locomotor pattern comparable to that of the intact animals. On the contrary, T9 transplanted animals developed limited and disorganized movements as those of nontransplanted animals. The correlation of the locomotor performances with the level of reinnervation of the spinal cord suggests that serotonergic reinnervation of the L1-L2 level constitutes a key element in the genesis of this locomotor rhythmic activity. This is the first in vivo demonstration that transplanted embryonic raphe cells reinnervating a specific level of the cord activate a locomotor behavior.

Key words: locomotion; spinal rat; transplantation of embryonic neurons; locomotor recovery; kinematic analysis; EMG
Traumatic lesions of the spinal cord yield a loss of supraspinal control of motor functions and specifically a loss of voluntary locomotor activity, although the spinal cord contains the necessary circuitry to generate the basic locomotor pattern (Grillner, 1981). This central pattern generator (CPG) can generate the locomotor command of each muscle of the limbs, set the rhythm of locomotor cycles, and insure intralimb and interlimb muscular coordinations. The CPG is modulated by supraspinal descending inputs and sensory afferents (Rossignol et al., 1988). After the loss of supraspinal control, there are therefore some residual motor functions that are more or less re-expressed as a function of species (Grillner, 1981; Rossignol et al., 1996; Rossignol et al., 2000). For instance, spinal cats recuperate some spontaneous hindlimb locomotor functions (Rossignol, 1996), whereas spinal rats only develop some uncoordinated flexion and extension

\footnotetext{
Received Feb. 10, 2000; revised April 5, 2000; accepted April 13, 2000.

This work was supported by a France-Québec Exchange Program, Conseil de Recherches Médicales du Canada, IRME, Association Francaise Contre les Myopathies, VERTICALE, and BIOMED. We thank J. R. Teilhac for artwork and M. Lahsini for secretarial assistance.

Correspondence should be addressed to Dr. Minerva Gimenez y Ribotta, Institut National de la Santé et de la Recherche Médicale, U 336, Université Montpellier II, Pl. E. Bataillon, CC 106, F-34095 Montpellier, France. E-mail: mgyr@univmontp2.fr.

Copyright (C) 2000 Society for Neuroscience $\quad 0270-6474 / 00 / 205144-09 \$ 15.00 / 0$
}

movements (Yakovleff et al., 1989; Broton et al., 1996; McDonald et al., 1999).

To improve locomotor recovery after spinal cord injury three experimental strategies have been developed (Giménez y Ribotta and Privat, 1998): (1) Neuroprotection soon after injury to reduce the progressive secondary injury processes (Pencalet et al., 1993; Gaviria et al., 2000). (2) Promotion of axonal regeneration by trophic factors or by acting on inhibitors of the regeneration or by cell or/and gene therapy to reestablish the supraspinal inputs (Goldberger et al., 1993; Iwashita et al., 1994; Giménez y Ribotta et al., 1995; Bregman et al., 1997; Grill et al., 1997; Cheng et al., 1997; Li et al., 1997; Ramón-Cueto et al., 1998). (3) Activation of the sublesional spinal cord circuitry.

In this third strategy the approaches are directed (1) at enhancing the influence of sensory afferents on CPG, through locomotor training, (Barbeau and Rossignol, 1987; De Leon et al., 1998), or/and (2) at restituting partially the missing neurotransmitters by supplying (intravenously, intraperitoneally, or intrathecally) agonists of monoamines depleted in the cord because of the lesion. This approach is founded on the role played by monoaminergic descending systems in the modulation of locomotion (Lundberg, 1966; Grillner and Dubuc, 1988; Jacobs and Fornal, 1993; Gerin and Privat, 1998).

We have developed an original approach in a cord-transected rat model. Our purpose was to supply the sublesional spinal cord 
with missing monoaminergic supraspinal afferents by transplanting embryonic brainstem neurons (Privat et al., 1986, 1988, 1989). Our previous studies have indeed shown that locus coeruleus or raphe cells transplanted in the sublesional cord can reinnervate specific targets (Privat et al., 1988; Rajaofetra et al., 1992; Giménez y Ribotta et al., 1996), restore the lesion-induced increase in receptor densities of neurotransmitters (Roudet et al., 1995), promote hindlimb weight support, and trigger a locomotor activity on a treadmill in animals without any other pharmacological treatment or training (Yakovleff et al., 1995; Feraboli-Lohnherr et al., 1997; Giménez y Ribotta et al., 1998). This functional recovery of posture and locomotion has recently been challenged in a model of spinal rat transplanted with mice stem cells (McDonald et al., 1999). However, only partial weight support was achieved, thus underlining the necessity of precise evaluation of locomotor behavior to claim restoration of function.

In the present study, we have transplanted embryonic raphe cells at two different levels of the sublesional cord (T9 and T11), and then performed an analysis of the kinematic and EMG activity synchronously recorded during locomotion. Finally, with the aim of discriminating whether the action of serotonin on CPG is associated to a specific and precise level of the cord, a systematic anatomofunctional correlation was established between the reinnervated level of the cord and the locomotor performance in both groups of transplanted animals compared to that of intact and transected nontransplanted animals.

\section{MATERIALS AND METHODS}

Animals. Eighteen adult female Sprague Dawley rats weighing 220-250 gm (IFFA Credo, Arbresle, France) were divided in four groups: intact $(n=3)$, transected $(n=5)$, transected/transplanted at T11 $(n=5)$, and transected/transplanted at T9 $(n=5)$. To have similar biomechanic constraints exerted on hindlimbs at the beginning and the end of the experiment ( 2 months), we used only females because their initial weight was not modified over this period. Animals were cared for and surgically handled in accordance with the European Community Council Directive (24 November 1986, 86/609/EEC).

Spinal cord transection. Animals were deeply anesthetized with Equithesin $(3 \mathrm{mg} / \mathrm{kg}$, i.p.) and underwent a laminectomy at the spinal thoracic level T8. The spinal cord and the dura were completely transected with ophthalmic microscissors. After hemostasis, the back musculature was sutured. Animals were placed in a special heated box until full postoperative recovery. This surgery was followed by $8 \mathrm{~d}$ of prophylactic antibiotic administration to prevent staphylococcic infection (Oxacillin $0.3 \mathrm{mg} / 100 \mathrm{gm}$, i.p.) and urinary infections (Gentamycine $0.2 \mathrm{mg} / 100 \mathrm{gm}$, i.p.). The bladder was emptied manually $2-3$ times daily until reflex voiding was established (i.e., after $\sim 10 \mathrm{~d}$ ).

Implantation of electrodes. During the same surgery, wire electrodes were chronically and bilaterally implanted (Chau et al., 1998b) in several flexor and extensor muscles: ilio-psoas (Ip) hip flexor, semitendinous knee (St) flexor, tibialis anterior (TA) ankle flexor, vastus lateralis (VL) knee extensor, and gastrocnemius medialis (GM) ankle extensor for chronic EMG recording during treadmill locomotion.

Cell suspension. The embryonic donor tissue was taken from the same inbred strain as the host animals. Embryos were taken after laparotomy from pregnant rats at embryonic day 14 (E14). The day after mating was considered day 0 . The microdissection of the tissue has been previously described in detail (König et al., 1989). Briefly, the caudal rhombencephalon, extending from pontine flexure to the cervical end of the spinal cord and containing the B1-B3 raphe nuclei, was dissected out in HBSS (Life Technologies, Gaithersburg, MD). After mechanical dissociation by gentle pipetting in calcium/magnesium-free Puck's solution (Life Technologies), the suspension was centrifuged at $80 \times g$ for $10 \mathrm{~min}$, resuspended in minimal essential culture medium (Life Technologies), and adjusted to a final concentration of 50,000 cells $/ \mu l$.

Grafting. Animals were transplanted 1 week after transection following the procedure described earlier (Rajaofetra et al., 1992), because this period is optimal for graft development. Briefly, a second laminectomy was performed at the T9 or at the T11 spinal level, and $4 \mu \mathrm{l}$ of the cell suspension was injected into the spinal cord $(1 \mathrm{~mm}$ below the pial surface) with a metallic needle $(0.4 \mathrm{~mm}$ diameter $)$ connected to a Hamilton microsyringe. The needle was withdrawn 2 min after the end of the injection to avoid suspension reflux. The musculature was then sutured, and the animals were treated as described above.

Kinematic and EMG analysis. After a survival of 2 months the animals were submitted to a kinematic and EMG analysis. Six light-reflecting spots ( $3 \mathrm{~m}$ material) were glued on the joints of animals (Fig. $1 A$ ), and movements during treadmill locomotion (speed, 0.1 or $0.2 \mathrm{~m} / \mathrm{sec}$ ) were recorded on video using a video camera with an electronic shutter $(1 / 1000 \mathrm{sec})$. The EMG signals were synchronously recorded on a 14 channel analog Honeywell recorder at $13.75 \mathrm{~cm} / \mathrm{sec}$, which gave a frequency response of $1250 \mathrm{~Hz}$. A Standard Motion Picture and Television Engineering (SMPTE) time code was simultaneously recorded on the analog and video tapes for off-line synchronization of kinematic and EMG data.

For kinematic analysis, a field by field analysis (60 fields/sec) giving a $16.6 \mathrm{msec}$ resolution between frames was performed on selected video sequences using a Peak Performance System two-dimensional analysis system for detecting the $x-y$ coordinates of the light reflecting spots (Fig. $1 A$ ). These coordinates were then used to reconstruct the movements in the form of stick diagrams (Fig. $2 A, D$ ) or angular joint displacements (Fig. 2B,E).

Retransection of the spinal cord. After analysis of the locomotor performance, two transected/transplanted in T11 animals underwent a second cord transection at the same T8 level, to eliminate any possible influence of supraspinal regenerating axons on the locomotor activity. Their locomotor movements were again documented after this second transection.

Immunohistochemistry. After the kinematic and EMG analysis, animals were anesthetized with sodium pentobarbital $(50 \mathrm{mg} / \mathrm{ml})$ and intracardially perfused with $5 \%$ glutaraldehyde in 50 mm sodium metabisulphite $/ 50 \mathrm{~mm}$ cacodylate buffer, $\mathrm{pH} 7.5$. Spinal cords were removed and post-fixed in the same fixative for $24 \mathrm{hr}$ at $4^{\circ} \mathrm{C}$. The cord was dissected, and serial transverse vibratome sections $(50 \mu \mathrm{m})$ were performed throughout the graft and at L1-L2 level. Longitudinal sections from spinal cord segments between graft and L1-L2 levels were also performed to evaluate the decreased reinnervation from the transplantation site. Then, all sections were processed for serotonin (5-HT) immunodetection. After treatment with trypsin-EDTA (0.25\%; Life Technologies) for $5 \mathrm{~min}$ and $10 \mathrm{~mm}$ sodium borohydride for $10 \mathrm{~min}$, sections were successively incubated with a rabbit polyclonal antibody against 5-HT (1:10,000; kindly provided by Dr. Geffard) with $1 \%$ nonspecific goat serum in Tris-sodium metabisulfite buffer for $48 \mathrm{hr}$ at $4^{\circ} \mathrm{C}$. After rinses in $50 \mathrm{~mm}$ Tris-saline buffer, $\mathrm{pH} 7.5$, sections were successively incubated in goat anti-rabbit antiserum and rabbit peroxidase antiperoxidase, both diluted 1:100 in Tris saline buffer containing 1\% nonspecific goat serum for $30 \mathrm{~min}$ at room temperature. Immunoreactivity was revealed with $0.1 \% 3,3^{\prime}$-diaminobenzidine in the presence of the $\mathrm{H}_{2} \mathrm{O}_{2}$.

\section{RESULTS}

\section{Kinematic and EMG analysis}

To compare locomotor performance of different groups of animals to a reference activity, locomotor movements of the hindlimbs of intact animals were analyzed. A sequence of five frames taken from a step cycle of an animal walking at $0.1 \mathrm{~m} / \mathrm{sec}$ is illustrated in Figure $1 A$. The tail of the animal was lightly held to help maintain a constant speed and provide the same sensory stimulus as was performed for all animal groups. The kinematic and EMG analyses are shown in Figure $2 A-C$ to compare with T11 transplanted animals in Figure $2 D-F$.

All the transected nontransplanted animals (three of three) moved their forelimbs, whereas their hindquarters dragged along the ground with the joints in extension. Some flexion and extension movements were occasionally developed and often alternated in both hindlimbs. Tail pinching and movement of the treadmill elicited rare and weak movements limited to proximal joints without rhythmic activity, as shown in the sequence of frames of Figure $1 B$. Such sequences were not further analyzed.

All the T11 transplanted animals were able to stand up supporting their body weight and to walk on the treadmill with tail 


\section{A Intact}

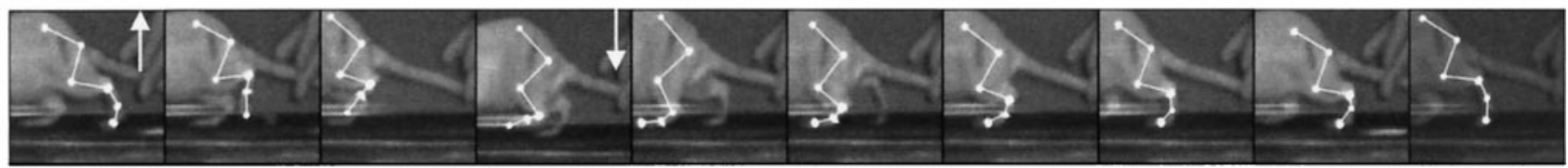

B Spinal T8 - Non transplanted

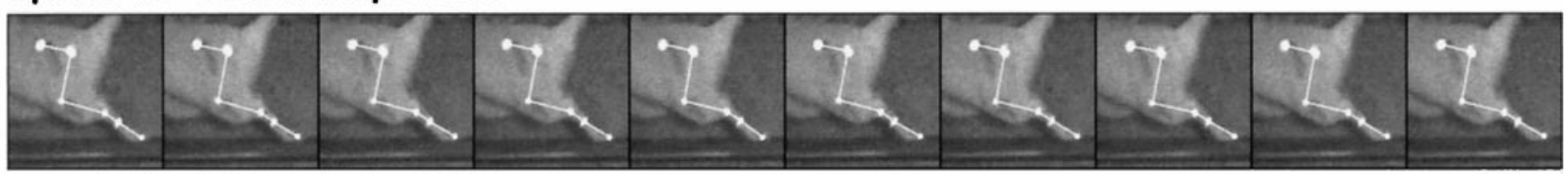

C Spinal T8 - Transplanted T11

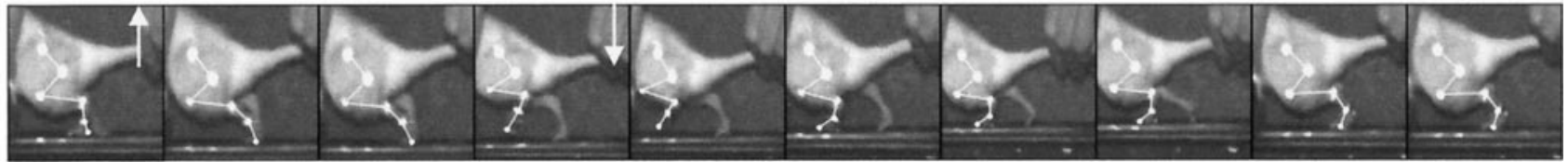

D Spinal T8 - Transplanted T11 - Re-sectioned T8

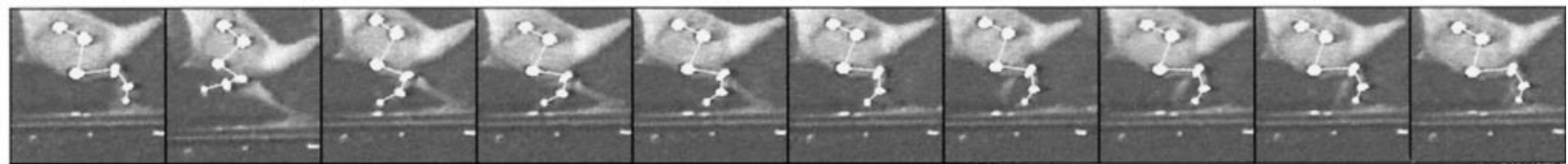

E Spinal T8 - Transplanted T9

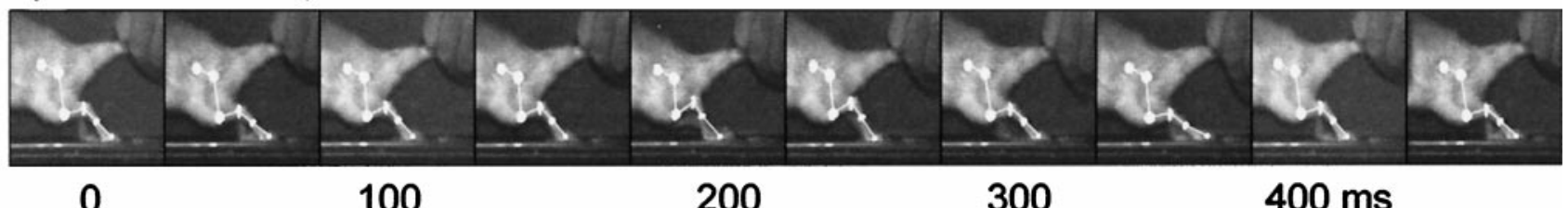

Figure 1. Sequences of selected video frames in five different conditions. Frames were grabbed using a video grabber at approximately every 50 msec starting at foot lift of one step sequence (beginning of swing) on the left side. The spots are the light reflecting markers placed on the left leg. The white lines were added post hoc to facilitate the visualization of angular movements as shown in figures such as Figure $2 A$. Times are approximations to the closest 50 msec. Upward and downward facing arrows indicating foot lifts and foot contacts have been added when appropriate. A, An intact adult rat; $B$, A spinal adult rat 10 weeks after transection; $C$, A T11-transplanted rat ( 9 weeks after grafting); $D$, The same animal as in $C$ (10 weeks after grafting) after a new transection at T8 spinal cord level. E, A T9-transplanted rat (9 weeks after grafting).

pinching. Locomotor movements of the hindlimbs globally resembled those of intact animals (Fig. 1, compare $A, C$ ) as is also illustrated by stick diagrams (Fig. 2A,D). Despite a more crouched posture, animals performed several complete step cycles with an appropriate swing, bringing the foot slightly in front of the hip and making a plantar foot contact for the rest of the stance phase until the next swing. The locomotor sequences elicited by tail stimulation lasted as long as the stimulation (routinely $\sim 20 \mathrm{sec}$ corresponding to 20-30 successive step cycles). In absence of tail stimulation, the animals appeared unable to raise spontaneously their hindquarters and were lying on one side. In that case, treadmill stimulation could evoke some alternated movements in hindlimbs without proper foot contact with the ground. When placed on the treadmill in a convenient posture for locomotion by the experimenter, these T11 transplanted animals could stand up on their hindlimbs. However, this posture could not be maintained for more than few seconds (3-5) without help, stressing the fact that the control of balance in hindlimbs remained largely impaired, in accordance with the fact that the spinal cord remained disconnected from supraspinal structures despite the transplantation (see below, the effects of retransection of the spinal cord).
We performed a detailed comparative kinematic and EMG analysis between intact and T11 transplanted animals as illustrated in Figure 2 in two examples representative of all the animals of each group. During the swing phase, intact animals showed a coordinated ankle, knee, and hip flexion that elevates the foot to bring it upward and forward (Fig. 2A), exhibiting characteristic angular excursions (Fig. 2B). In T11 transplanted animals the foot was not brought forward as much as in intact animals (Fig. 2D) because the angular range was biased toward extension (Fig. 2E). Probably as a consequence of changes in biomechanical constraints linked to this overextension, the angular traces showed less yield of the knee and ankle after foot contact than in intact animals. However, the gradual extension of the ankle joint observed in the stick diagram (Fig. 2D) indicated that the limb is not simply dragged backward by the treadmill, but that the foot actively pushes against ground. The corresponding synchronized EMG pattern showed, in intact animals, discharges of iIp, iSt, and iTA during the swing phase and that of iVL and iGM during stance phase (Fig. 2C). The contralateral St (coSt) also discharged during the ipsilateral stance, indicating a good alternation between limbs (Fig. 2C). In T11 transplanted animals the EMG pattern was not very different from that observed in 


\section{INTACT}

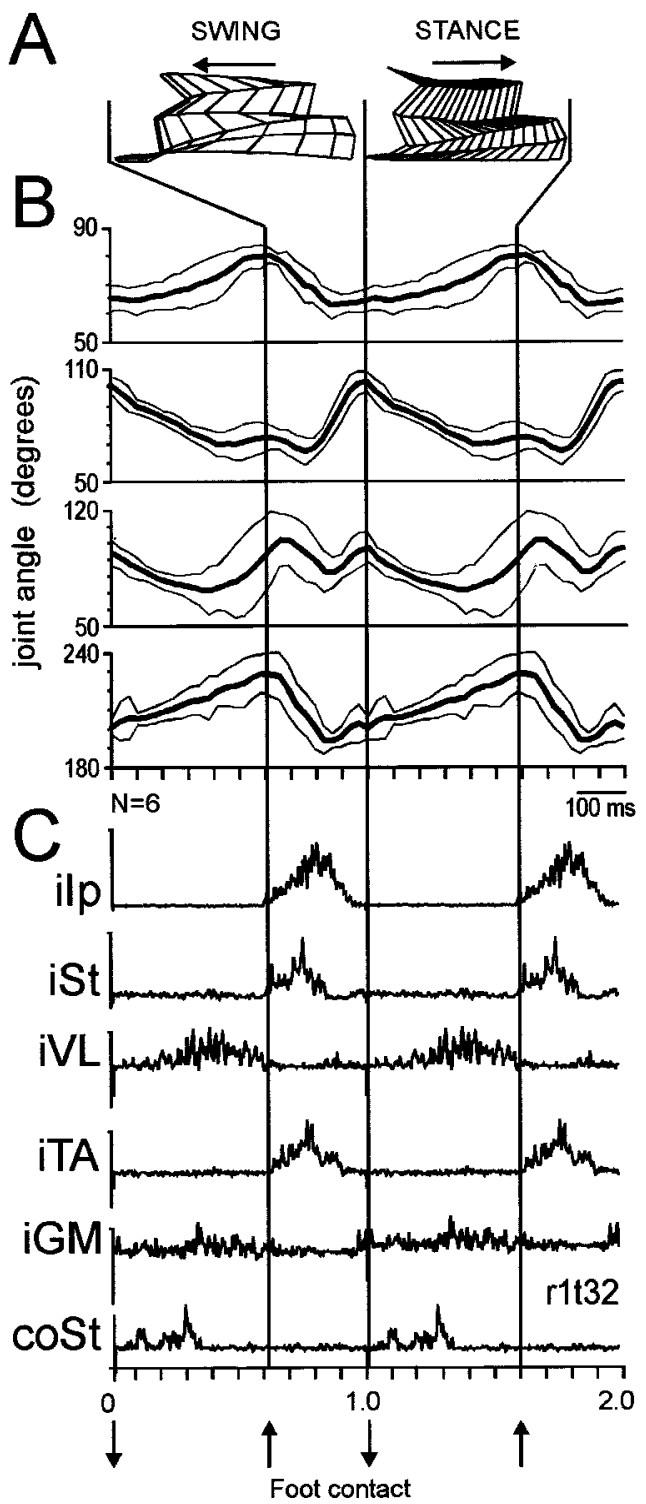

SPINAL T11-TRANSPLANTED

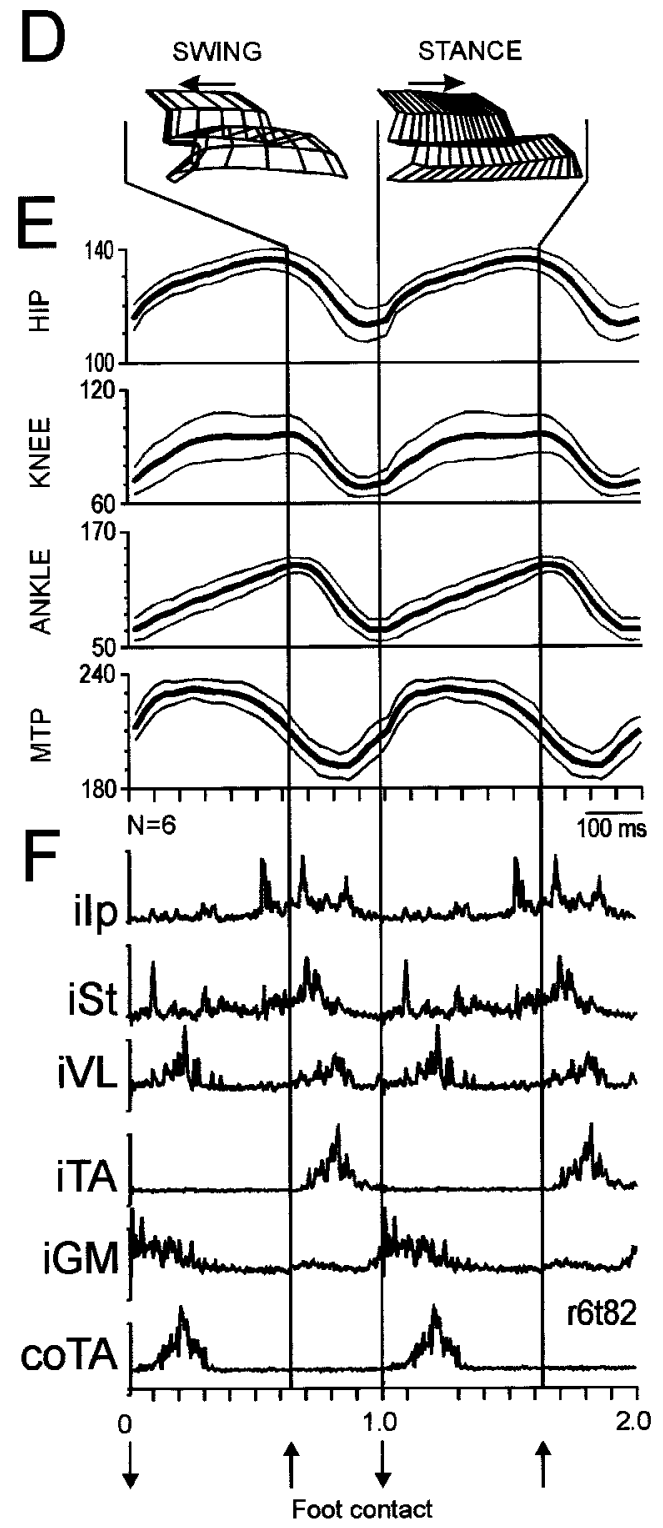

Figure 2. Comparison of rhythmic locomotor activity in an intact rat $(A-C)$ and a T11 animal 9 weeks after transplantation of embryonic raphe cells from E14 embryos $(D-F) . A, D$, Reconstruction, as stick diagrams, of treadmill locomotor movements during swing and stance phases. Each stick figure is displaced from the previous by an amount equivalent to the foot displacement to avoid overlap of all the figures. $B, E$, Variations of mean angle joints (thick lines) and their SDs (thin lines) from six consecutive step cycles, in (from top to bottom) hip, knee, ankle, and metatarsophalangeal (MTP) joints. The same normalized step cycle is displayed twice to facilitate viewing the events at around the trigger point (foot contact of the limb facing the camera, downward facing arrow). The foot lift of the same limb is also indicated at the bottom of the figure by upward facing arrows. Angular excursion of various joints are averaged for six cycles and synchronized on foot contact. $C, F$, Corresponding synchronized EMG activity in various muscles of ipsi ( $i$ ) or contralateral (co) hindlimbs. Ip, Iliopsoas (hip flexor); St, semitendinosus (knee flexor and hip extensor); $V L$, vastus lateralis (knee extensor); $T A$, tibialis anterior (ankle flexor); GM, gastrocnemius medialis (ankle extensor). Note the discharges of iIp, iSt, and iTA during the swing phase and that of iVL and iGM during stance. The contralateral St (coSt) also discharges during the ipsilateral stance, indicating a good alternation between limbs.

intact animals (Fig. 2, compare $C, F$ ). However, two slight differences were observed in proximal muscles. First, the bursting in flexor muscles iIP and iSt appeared sometimes less well shaped in T11 transplanted animals compared to intact animals (Fig. $2 F$ ). This deficit in flexor activity could account for the general overextended position of the limb. Second, some extra bursts could be observed in the knee extensor muscle (VL) during the swing phase which could contribute, together with the overextended position of the limb, to the diminution of the yield.

The relationship between the burst duration of the flexor TA and the extensor GM is illustrated as a function of cycle duration (Fig. 3). For intact animals (Fig. 3A), which could maintain a regular locomotor rhythm, the treadmill speed was varied from 0.1 to $0.5 \mathrm{~m} / \mathrm{sec}$ to record the EMG activity and the movement for various step cycle durations. In transplanted walking animals, flexor muscles discharged a burst of relatively fixed duration during swing phase, whereas the burst of extensor muscle during stance phase varied with the cycle duration, discharging for longer periods at lower speeds when cycle durations are longer; the same has been shown in intact rats in this study as well as by 


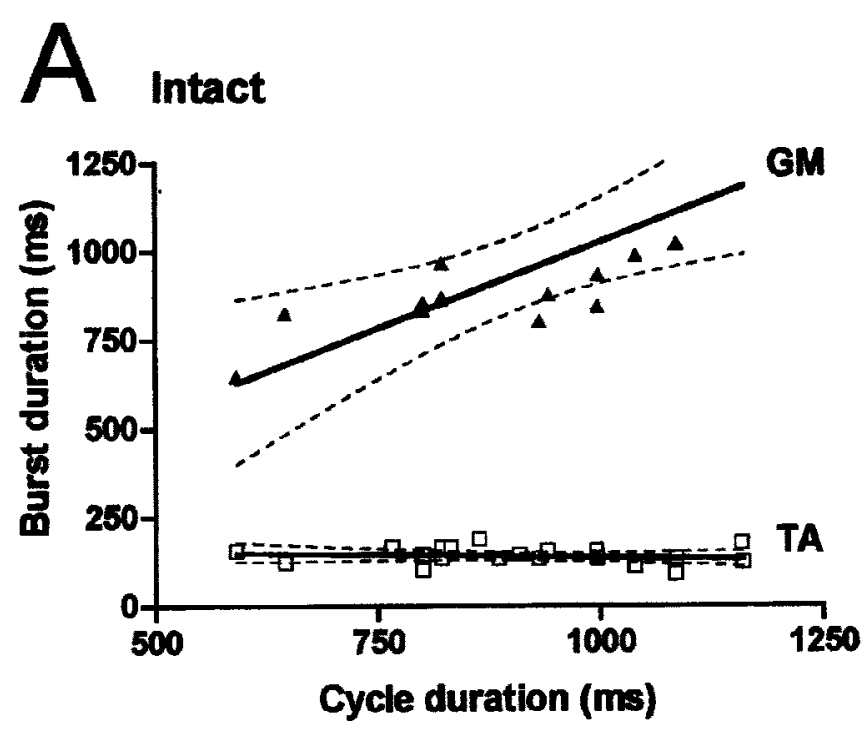

\section{B Transplanted T11}

Figure 3. Relationships between duration of extensor (GM for gastrocnemius medialis) or flexor (TA for tibialis anterior) EMG bursts in intact ( $A$ ) or transplanted $(B)$ adult rats 10 weeks after spinal cord transection. $A$, Intact rat. $n=21 ; r^{2}=0.035$; slope $=-0.30 \pm 0.036$ for TA muscle and $n=14$, $r^{2}=0.87$; slope $=0.98 \pm 0.30$ for GM muscle. $B$, Spinal rat (T8) transplanted (T11) for 10 weeks. $n=12 ; r^{2}=0,091 ;$ slope $=-0.051 \pm 0.051$ for TA and $n=12 ; r^{2}=0.83$; slope $=0.75 \pm 0.11$ for GM.

others (Gruner and Altman, 1980; Nicolopoulos-Stournaras and Iles, 1984), and also in intact cats (Grillner, 1981), suggesting that transplanted animals can reexpress a locomotor pattern with a structure similar to that of the intact animals.

After a second transection at T8, the T11 transplanted animals maintained their locomotor performance as before, and this is illustrated in Figure $1 D$.

T9 transplanted animals did not stand up, and, as nontransplanted animals, were unable to support the hindquarters. The feet dragged on the treadmill belt with all the hindlimb joints in extension. With tail stimulation, some small ankle movements were occasionally generated alternating in both hindlimbs, but angular excursions were very limited, remaining almost always in the extension range (Fig. $1 E$ ). Angle variations of the toes were out of phase with proximal joints, probably because these movements were passive. Rhythmic movements and their correlative EMG patterns were so disorganized that they could not be averaged, and consequently, they are not illustrated. A struggling reaction could also be elicited by pinching the toes (Pearson and Rossignol, 1991).

\section{Immunohistochemical analysis}

The kinematic and EMG analyses were correlated with a blind postmortem immunohistochemical study of the 5-HT reinnervation in the sublesional spinal cord.

As previously reported (Björklund and Skagerberg, 1982), in transected nontransplanted animals, the spinal cord at sublesional level was totally devoid of 5-HT immunoreactivity, as expected from the degeneration of all descending inputs, whereas above the section the 5-HT innervation pattern remained similar to that of intact animals, i.e., 5-HT immunoreactivity was distributed in the main target regions, the dorsal horn, the intermediolateral column, the ventral horn around motoneurons, and in proximity of the central canal.

In T11 transplanted animals, 2 months after grafting, the spinal cord exhibited a transplant usually located close to the midline with many 5-HT-immunoreactive perikarya (Fig. $4 B$ ). In the transplants, serotonergic neurons represented $\sim 2-4 \%$ of trans- planted cells, and their rate of survival appears superior to the mean survival of transplanted cells (Privat et al., 1988). An extensive network of immunoreactive varicose processes was developed from the transplant which extended out in all directions, occasionally crossed the midline, reinnervating specific targets. On longitudinal sections, 5-HT-immunoreactive fibers with abundant varicosities were observed running longitudinally and extending rostrally and caudally close to the central canal. Thin fibers were also observed distributed in the intermediolateral column, some of them crossing perpendicularly toward the central canal. Moreover, in the ventral horn a preferential 5-HT innervation was observed around motoneurons. This innervation became gradually sparser at increasing distance from the area of the transplant, but was well detectable up to L1-L2 level $(8 \mathrm{~mm}$ caudally to the transplant) (Fig. 4C). Some 5-HT fibers were also occasionally detected in the white matter.

In T9 transplanted animals, 2 months after grafting, the spinal cord exhibited, as in T11 animals, a well developed transplant with many 5-HT-immunoreactive perikarya. Grafted neurons gave rise to an extensive network of immunoreactive fibers (Fig. $4 E$ ), which bilaterally reinnervated specific targets. Longitudinal sections showed numerous 5-HT fibers from the transplant, which extended rostrally and caudally, and some of them crossed perpendicularly toward the central canal. Again, the reinnervation of the ventral horn showed a preferential concentration around of motoneurons. This innervation became gradually sparser at some distance from the transplant, and scarcely reached the T13 level. An important difference with T11 transplanted animals is that no 5-HT fibers were detected at the L1-L2 levels in T9 transplanted animals (Fig. 4F).

\section{DISCUSSION}

This study demonstrates that kinematic and EMG patterns of the movement expressed by T11 transplanted animals correspond, contrary to those of $\mathrm{T} 9$ transplanted animals, to a well defined locomotor pattern comparable to that of the intact animals. Moreover, the correlation of the locomotor performance with the 

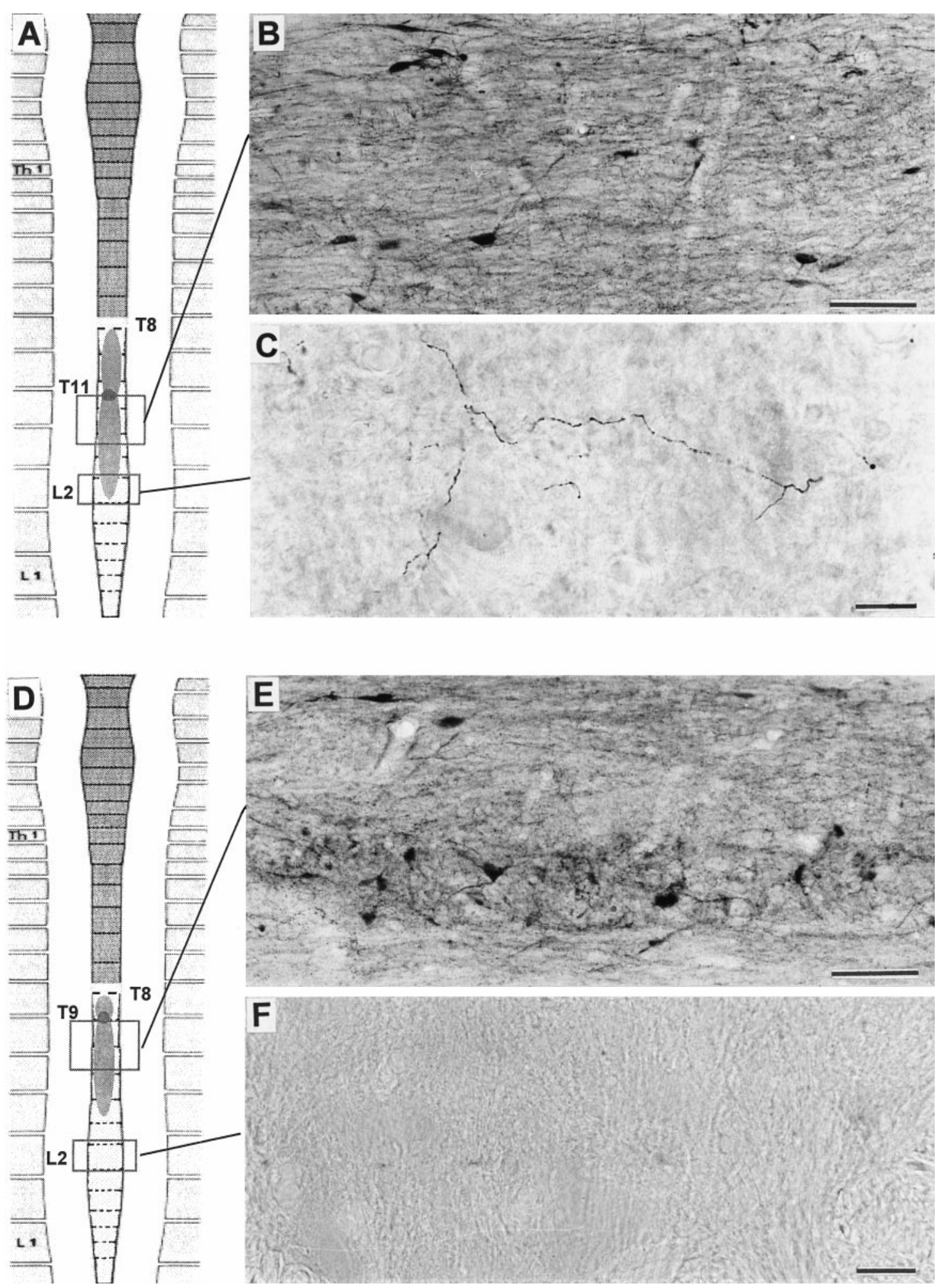

Figure 4. Diagrams and immunocytochemical detection of serotonin (5-HT) in vibratome sections of the spinal cord. $A$, Diagram illustrating the spinal cord in a T11 animal and the segments taken for immunocytochemical detection of 5-HT as seen in micrographs $B$ and $C$. $B$, A longitudinal rostrocaudal section through the transplant area showing ovoid and multipolar 5-HT-immunoreactive neurons with thin and varicose processes distributed in the gray as well as white matter. Scale bar, $100 \mu \mathrm{m}$. $C$, A transverse section at L2 level that shows very varicose 5-HT fibers distributed in the ventral horn. Scale bar, $25 \mu \mathrm{m}$. D, Diagram illustrating the spinal cord in a T9 animal and segments taken for immunocytochemical detection of 5-HT as seen in micrographs $E$ and $F$. E, A longitudinal rostrocaudal section through the transplant area showing, as in T11 animals, 5-HT-immunoreactive neurons with a dense immunoreactive neuropil. Scale bar, $100 \mu \mathrm{m}$. F, A transverse section at L2 level where no 5-HT-immunoreactive fibers can be detected. Scale bar, $25 \mu \mathrm{m}$. 
level of reinnervation of the spinal cord allows to suggest that 5-HT reinnervation of the upper lumbar segments (namely L1L2) constitutes a key element for the expression of the locomotor rhythmic activity. This is the first in vivo demonstration that transplanted embryonic raphe cells to the spinal cord reinnervating a specific level can promote the locomotor activity in adult spinal rats.

At present, the generation of locomotion within the spinal cord by a specialized network, the so-called CPG, is clearly established (Grillner, 1981; Rossignol, 1996). In the absence of supraspinal descending inputs, this circuitry becomes ineffective, especially when there is insufficient activation by peripheral afferent inputs. Although supraspinal descending sensory stimulation and treadmill training pathways appear to contribute to plastic mechanisms yielding to the recovery of some parameters of locomotion in spinal cats (Barbeau et al., 1987; Beattie et al., 1993; Helgren and Goldberger, 1993; Rossignol et al., 1996; De Leon et al., 1998), such a recovery appears absent in rats (Yakovleff et al., 1995; Broton et al., 1996; McDonald et al., 1999). Adult spinal rats maintain the hindlimbs passively extended and can only develop some nonlocomotor rhythmic activities such as air stepping (Meisel and Rakerd, 1982), fictive paw-shaking (Yakovleff et al., 1995), or some other more disorganized movements (FeraboliLohnherr et al., 1997).

An approach designed to activate the CPG has been to supply the sublesional cord with missing monoaminergic descending afferents either by pharmacotherapy (Chau et al., 1998a) or by using a strategy of transplantation with embryonic brainstem neurons (Privat et al., 1988).

Pharmacological treatments with monoaminergic agonists can trigger locomotor activity in chronic spinal cats for a few hours (Barbeau and Rossignol, 1991). Noradrenergic agonists can initiate locomotion and markedly increase the step cycle and flexor muscle duration (Chau et al., 1998b), whereas serotonergic agonists increase the duration and the amplitude of both flexor and extensor EMGs (Barbeau and Rossignol, 1990, 1991). Pharmacological treatments have also been combined together with locomotor training to enhance the recovery of locomotion in spinal cats (Rossignol et al., 1986, 2000; Barbeau et al., 1987).

In the present study we used a previously described strategy of transplantation aimed at permanently supplying the sublesional spinal cord with the missing neurotransmitters (Privat et al., 1988). After the complete transection of the thoracic spinal cord, the sublesional cord is totally devoid of supraspinal descending monoaminergic systems (Haggendal and Dahlstrom, 1973; Magnusson, 1973). This constitutes an optimal environment for analyzing the influence of transplanted serotonergic raphe cells at two different levels on locomotor activity. The modulatory role of serotonin in setting the level of excitability appropriate for CPG function has been demonstrated during treadmill-induced locomotion in spinal cats (Barbeau and Rossignol, 1991), but also during undisturbed locomotion by electrophysiological analysis of serotonergic neurons (Jacobs and Fornal, 1993) or by direct evaluation of the release of serotonin in running rats permanently implanted with microdialysis probes (Gerin et al., 1995). Furthermore, the stimulation of the CPG by serotonin has been widely documented in an in vitro spinal cord preparation of neonate rats (Cazalets et al., 1992). This role of serotonin, facilitator of motor output, is also supported by its extensive plasticity after modifications of targets (Marlier et al., 1991a,b, 1992; Poulat et al., 1992).

The present anatomical results confirm our previous studies using the same paradigm. Transplanted serotonergic neurons specifically reinnervated the main target areas in the sublesional cord with an innervation pattern similar to that of intact animals (Privat et al., 1988; Rajaofetra et al., 1992; Yakovleff et al., 1995; Feraboli-Lohnherr et al., 1997; Dumoulin et al., 2000), independently of the transplanted level.

Kinematic and EMG analysis were mandatory to discriminate between uncoordinated movements and genuine locomotion, characterized by correlated burst discharges with angular excursions of movements. Such a correlation has never been yet performed in adult rats using this paradigm. Indeed in their most recent report, McDonald et al. (1999) describe a functional recovery after partial lesion and transplantation of stem cells, which is only based on a 2 point recovery on the BBB score. This cannot be taken as a significant landmark of locomotion. In our experiments, first, the posture adopted by T11 transplanted animals demonstrated bilateral foot placement on the plantar surface and their ability to support the body weight, contrasting with T9 animals, which resembled, in that respect, to transected nontransplanted animals. Second, a rhythmic activity with cycle duration within locomotor range was induced in T11 animals, without any pharmacological treatment or training. This was altogether absent in T9 animals, which again resembled more the transected and nontransplanted animals. Third, coordinated bursts of flexor and extensor muscles were respectively produced during the swing and stance phases, which allowed to move the foot upward and forward, alternating with bursts of homologous contralateral limb muscles. These results, supplemented now by a correlative kinematic analysis, confirm again our previous EMG studies using the same paradigm (Yakovleff et al., 1995; FeraboliLohnherr et al., 1997).

However, interestingly, the present study demonstrates that transplanted animals can exhibit also a nonlocomotor rhythmic activity with invariable burst durations and limited movements, if the reinnervated level of the cord is not appropriate. In T9 animals, serotonergic reinnervation pattern did not proceed below T13 cord level, whereas serotonergic fibers reached L1-L2 level in T11 animals. This suggests that reinnervated L1-L2 segments play a specific role in the nervous control of locomotion compared to more rostral segments. This point is particularly interesting, because a similar hypothesis has already been suggested in an in vitro model of neonatal rats (Cazalets et al., 1995). This also provides further support to the notion that the upper lumbar segments are more excitable and can lead locomotion (Kjaerulff and Kiehn, 1996). However, the activation of lower thoracic segments does not appear sufficient, and the activation of upper lumbar spinal cord (L1-L2) at least is necessary to induce locomotion. The activating role of transplanted serotonergic neurons in this locomotor behavior has been previously demonstrated (Feraboli-Lohnherr et al., 1997). Recent studies (J. R. Cazalets, personal communication) suggest that a locomotor pattern can be evoked from lower lumbar/sacral level, but under the control of upper lumbar cord.

Other studies have shown restoration of some aspects of locomotion in spinal transplanted rats. These studies aimed, however, at reconnecting the two stumps of the cord after injury by transplantation, at the injury site, of embryonic spinal cord, peripheral nerves, or particular glial cells as well as other genetically modified cells (Kunkel-Bagden and Bregman, 1990; Iwashita et al., 1994; Cheng et al., 1997; Grill et al., 1997; Li et al., 1997; Ramón-Cueto et al., 1998; Chauvet et al., 1998; Prieto et al., 2000). In most of these studies, an important serotonergic regen- 
eration was observed, probably because of the high plasticity of this descending system (Marlier et al., 1991a, 1992; Poulat et al., 1992) favored by trophic factors derived or synthesized by the transplants or by providing permissive molecules to regenerating fibers. In any case, intrinsic circuits to the sublesional spinal cord are rarely taken in consideration for the recovery of function, and are as important as the factors provided by the transplants. Similarly, the restoration of locomotion is based on improvement of composite scores, which do not explore specifically the landmarks of locomotion exemplified in the present study. Thus, in the latter, the possibility of regenerating descending axons was eliminated after retransection of the cord in two T11 animals. The unaltered locomotor performance after retransection clearly indicates that the locomotor activity observed in our transplanted animals is attributable to the activation of lumbar spinal cord by serotonergic transplants.

A recent study in transected neonatal rats (Kim et al., 1999) that were transplanted, at the injury site, with embryonic spinal cord, then trained and pharmacologically treated with serotonergic agonists, has shown that these drugs improved significantly the motor performance of transplanted animals, and surprisingly, not of spinal rats. These results are at variance with our and other's results in adult spinal rats without any training (Barbeau and Bédard, 1981; Feraboli-Lohnherr et al., 1999) or cats (Barbeau and Rossignol, 1990). The authors suggested a permissive influence of the transplant on the action of serotonergic drugs and an interaction between both of influences in the remodeling of the circuitry. Although transected neonatal rats are known to exhibit a better transplant-mediated recovery of function than adult animals (Bregman, 1994), the modest serotonergic reinnervation observed in the latter experiment was not responsible for the restoration of motor function as ascertained by the lack of influence for reuptake inhibitors of serotonin. Moreover, the kinematic analysis in that study showed a large variability of patterns. Serotonergic agonists appeared to improve the motor performance in all animals; some spinal animals were comparable to the best transplanted animal, before agonist treatment. Finally, some transplanted animals exhibited no locomotor movement before drug treatment as do spinal animals (Kim et al., 1999).

In summary, our results demonstrate that serotonergic neurons transplanted in the spinal cord of adult rats, totally devoid of supraspinal descending inputs, are able to activate when they reinnervate a specific and precise level of the cord, the circuitry responsible for spinal pattern generation in adult paraplegic rats without any pharmacological treatment or training. Our hypothesis is that this precise serotonergic reinnervation in sublesional cord can provide an adequate supply of neurotransmitter in a specific site of the spinal cord, capable of activating the CPG and triggering a locomotor activity. These findings constitute the first step toward a reasoned transplantation strategy in spinal cordinjured patients.

\section{REFERENCES}

Barbeau H, Bédard PJ (1981) Similar motor effects of 5-HT and TRH in rats following chronic spinal transection and 5-7 dihydroxytryptamine injection. Neuropharmacology 20:477-481.

Barbeau H, Rossignol S (1987) Recovery of locomotion after chronic spinalization in the adult cat. Brain Res 412:84-95.

Barbeau H, Rossignol S (1990) The effects of serotonergic drugs on the locomotor pattern and on cutaneous reflexes of the adult chronic spinal cat. Brain Res 514:55-67.

Barbeau H, Rossignol S (1991) Initiation and modulation of the locomotor pattern in the adult chronic spinal cat by noradrenergic, serotonergic and dopaminergic drugs. Brain Res 546:250-260.
Barbeau H, Julien C, Rossignol S (1987) The effects of clonidine and yohimbine on locomotion and cutaneous reflexes in the adult chronic spinal cat. Brain Res 437:83-96.

Beattie MS, Leedy MG, Bresnahan JC (1993) Evidence for alterations of synaptic inputs to sacral spinal reflex circuits after spinal cord transection in the cat. Exp Neurol 123:35-50.

Björklund A, Skagerberg G (1982) Descending monoaminergic projections to the spinal cord. In: Brainstem control of spinal mechanisms (Sjolund B, Björklund A, eds), pp 55-88. Amsterdam: Elsevier.

Bregman BS (1994) Recovery of function after spinal cord injury: transplantation strategies. In: Functional neural transplantation (Dunnett SB, Björklund A, eds), pp 489-529. New York: Raven.

Bregman BS, Diener PS, McAtee M, Dai HN, James C (1997) Intervention strategies to enhance anatomical plasticity and recovery of function after spinal cord injury. Adv Neurol 72:257-275.

Broton JG, Nikolic Z, Suys S, Calancie B (1996) Kinematic analysis of limb position during quadrupedal locomotion in rats. J Neurotrauma 13:409-416.

Cazalets JR, Sqalli-Houssaini Y, Clarac F (1992) Activation of the central pattern generators for locomotion by serotonin and excitatory amino acids in neonatal rat. J Physiol (Lond) 455:187-204.

Cazalets JR, Borde M, Clarac F (1995) Localization and organization of the central pattern generator for hindlimb locomotion in newborn rat. J Neurosci 15:4943-4951.

Chau C, Barbeau H, Rossignol S (1998b) Early locomotor training with clonidine in spinal cats. J Neurophysiol 79:392-409.

Chau C, Barbeau H, Rossignol S (1998a) Effects of intrathecal alpha1and alpha2-noradrenergic agonists and norepinephrine on locomotion in chronic spinal cats. J Neurophysiol 79:2941-2963.

Chauvet N, Prieto M, Alonso G (1998) Tanycytes present in the adult rat mediobasal hypothalamus support the regeneration of monoaminergic axons. Exp Neurol 151:1-13.

Cheng H, Almstrom S, Gimenez-Llort L, Chang R, Ove Ogren S, Hoffer B, Olson L (1997) Gait analysis of adult paraplegic rats after spinal cord repair. Exp Neurol 148:544-557.

De Leon RD, Hodgson JA, Roy RR, Edgerton VR (1998) Locomotor capacity attributable to step training versus spontaneous recovery after spinalization in adult cats. J Neurophysiol 79:1329-1340.

Dumoulin A, Privat A, Giménez y Ribotta M (2000) Transplantation of embryonic raphe cells regulates the modifications of the GABAergic phenotype occurring in the injured spinal cord. Neuroscience 95:173-182.

Feraboli-Lohnherr D, Barthe JY, Orsal D (1999) Serotonin-induced activation of the network for locomotion in adult spinal rats. J Neurosci Res 55:87-98.

Feraboli-Lohnherr D, Orsal D, Yakovleff A, Giménez y Ribotta M, Privat A (1997) Recovery of locomotor activity in the adult chronic spinal rat after sublesional transplantation of embryonic nervous cells: specific role of serotonergic neurons. Exp Brain Res 113:443-454.

Gaviria M, Privat A, D'arbigny P, Kamenka JM, Haton H, Ohanna F (2000) Neuroprotective effects of gacyclidine after experimental photochemical spinal cord lesion in adult rats: dose-window and timewindow effects. J Neurotrauma 17:19-30.

Gerin C, Privat A (1998) Direct evidence for the link between monoaminergic descending pathways and motor activity: II. A study with microdialysis probes implanted in the ventral horn of the spinal cord. Brain Res 794:169-173.

Gerin C, Becquet D, Privat A (1995) Direct evidence for the link between monoaminergic descending pathways and motor activity. I. A study with microdialysis probes implanted in the ventral funiculus of the spinal cord. Brain Res 704:191-201.

Giménez y Ribotta M, Privat A (1998) Biological interventions for spinal cord injury. Curr Opin Neurol 11:647-654.

Giménez y Ribotta M, Rajaofetra N, Morin-Richaud C, Alonso G, Bochelen D, Sandillon F, Legrand A, Mersel M, Privat A (1995) Oxysterol (7 beta-hydroxycholesteryl-3-oleate) promote serotonergic reinnervation in the lesioned rat spinal cord by reducing glial reaction. J Neurosci Res 41:79-95.

Giménez y Ribotta M, Roudet C, Sandillon F, Privat A (1996) Transplantation of embryonic noradrenergic neurons in two models of adult rat spinal cord injury: ultrastructural immunocytochemical study. Brain Res 707:245-255.

Giménez y Ribotta M, Orsal D, Feraboli-Lohnherr D, Privat A (1998) Recovery of locomotion following transplantation of monoaminergic 
neurons in the spinal cord of paraplegic rats. Ann NY Acad Sci 860:393-411.

Goldberger ME, Murray M, Tessler A (1993) Graft and functional recuperation. Restor Neurol Neurosci 7:69-87.

Grill R, Murai K, Blesch A, Gage FH, Tuszynski MH (1997) Cellular delivery of neurotrophin-3 promotes corticospinal axonal growth and partial functional recovery after spinal cord injury. J Neurosci 17:5560-5572.

Grillner S (1981) Control of locomotion in bipeds, tetrapods and fish. In: Handbook of physiology (Brookhart JM, Mountcastle VB, eds), pp 1179-1236. Bethesda: Am Physiol Soc.

Grillner S, Dubuc R (1988) Control of locomotion in vertebrates: spinal and supraspinal mechanisms. In: Functional recovery in neurological disease (Waxman SG, ed), pp 425-453. New York: Raven.

Gruner JA, Altman J (1980) Swimming in the rat: analysis of locomotor performance in comparison to stepping. Exp Brain Res 40:374-382.

Haggendal J, Dahlstrom A (1973) The time course of noradrenaline decrease in rat spinal cord following transection. Neuropharmacology 12:349-354.

Helgren ME, Goldberger ME (1993) The recovery of postural reflexes and locomotion following low thoracic hemisection in adult cats involves compensation by undamaged primary afferent pathways. Exp Neurol 123:17-34.

Iwashita Y, Kawaguchi S, Murata M (1994) Restoration of function by replacement of spinal cord segments in the rat. Nature 367:167-170.

Jacobs BL, Fornal CA (1993) 5-HT and motor control: a hypothesis. Trends Neurosci 16:346-352.

Kim D, Adipudi V, Shibayama M, Giszter S, Tessler A, Murray M, Simansky KJ (1999) Direct agonists for serotonin receptors enhance locomotor function in rats that received neural transplants after neonatal spinal transection. J Neurosci 19:6213-6224.

Kjaerulff O, Kiehn O (1996) Distribution of networks generating and coordinating locomotor activity in the neonatal rat spinal cord in vitro: a lesion study. J Neurosci 16:5777-5794.

König N, Wilkie MB, Lauder JM (1989) Dissection of monoaminergic neuronal groups from embryonic rat brain. In: A dissection and tissue culture manual of the nervous system (Shahar A, Haber B, eds), pp 26-29. New York: Alan R. Liss.

Kunkel-Bagden E, Bregman BS (1990) Spinal cord transplants enhance the recovery of locomotor function after spinal cord injury at birth. Exp Brain Res 81:25-34.

Li Y, Field PM, Raisman G (1997) Repair of adult rat corticospinal tract by transplants of olfactory ensheathing cells. Science 277:2000-2002.

Lundberg A (1966) Integration in the reflex pathway. In: Nobel symposium: Muscular afferents and motor control (Granit R, ed), pp 179-224. Stokholm: Almquist and Wiksell.

Magnusson T (1973) Effect of chronic transection on dopamine, noradrenaline and 5-hydroxytryptamine in the rat spinal cord. Naunyn Schmiedebergs Arch Pharmacol 278:13-22.

Marlier L, Poulat P, Rajaofetra N, Privat A (1991a) Modifications of serotonin-, substance $\mathrm{P}$ - and calcitonin gene-related peptide-like immunoreactivities in the dorsal horn of the spinal cord of arthritic rats: a quantitative immunocytochemical study. Exp Brain Res 85:482-490.

Marlier L, Poulat P, Rajaofetra N, Privat A (1991b) Modifications of serotonergic immunoreactive pattern in the dorsal horn of the rat spinal cord following dorsal root rhizotomy. Neurosci Lett 128:9-12.

Marlier L, Poulat P, Rajaofetra N, Sandillon F, Privat A (1992) Plasticity of the serotonergic innervation of the dorsal horn of the rat spinal cord following neonatal capsaicin treatment. J Neurosci Res 31:346-358.

McDonald JW, Liu XZ, Qu Y, Liu S, Mickey SK, Turetsky D, Gottlieb DI, Choi DW (1999) Transplanted embryonic stem cells survive, differentiate and promote recovery in injured rat spinal cord. Nat Med 5:1410-1412.

Meisel RL, Rakerd B (1982) Induction of hindlimb stepping movements in rats spinally transected as adults or as neonates. Brain Res 240:353-356.
Nicolopoulos-Stournaras S, Iles JF (1984) Hindlimb muscle activity during locomotion in the rat (Rattus Norvegicus) (Rodent Muridae). J Zool Lond 203:427-440.

Pearson KG, Rossignol S (1991) Fictive motor patterns in chronic spinal cats. J Neurophysiol 66:1874-1887.

Pencalet P, Ohanna F, Poulat P, Kamenka JM, Privat A (1993) Thienylphencyclidine protection for the spinal cord of adult rats against extension of lesions secondary to a photochemical injury. J Neurosurg 78:603-609.

Poulat P, Marlier L, Pellas F, Rajaofetra N, Privat A (1992) Effects of the neonatal removal of superior cervical ganglion on serotonin and thyrotropin-releasing hormone immunoreactivity in the intermediolateral cell column of the rat spinal cord. Exp Brain Res 91:21-28.

Prieto M, Chauvet N, Alonso G (2000) Tanycytes transplanted into the adult rat spinal cord support the regeneration of lesioned axons. Exp Neurol 161:27-37.

Privat A, Mansour H, Pavy A, Geffard M, Sandillon F (1986) Transplantation of dissociated foetal serotonin neurons into the transected spinal cord of adult rats. Neurosci Lett 66:61-66.

Privat A, Mansour H, Geffard M (1988) Transplantation of fetal serotonin neurons into the transected spinal cord of adult rats: morphological development and functional influence. In: Transplantation into the mammalian CNS (Gash DM, Sladek JR, eds), pp 155-166. Amsterdam: Prog Brain Res Elsevier.

Privat A, Mansour H, Rajaofetra N, Geffard M (1989) Intraspinal transplants of serotonergic neurons in the adult rats. Brain Res Bull 22:123-129.

Rajaofetra N, König N, Poulat P, Marlier L, Sandillon F, Drian MJ, Geffard M, Privat A (1992) Fate of B1-B2 and B3 rhombencephalic cells transplanted into the transected spinal cord of adult rats: light and electron microscopic studies. Exp Neurol 117:59-70.

Ramón-Cueto A, Plant GW, Avila J, Bunge MB (1998) Long-distance axonal regeneration in the transected adult rat spinal cord is promoted by olfactory ensheathing glia transplants. J Neurosci 18:3803-3815.

Rossignol S (1996) Neural control of stereotypic limb movements. In: Exercise: regulation and integration of multiple systems (Rowell LB, Sheperd JT, eds), pp 173-216. Am Physiol Soc.

Rossignol S, Barbeau H, Julien C (1986) Locomotion of the adult chronic spinal cat and its modification by monoaminergic agonists and antagonists. In: Development and plasticity of the mammalian spinal cord (Goldberger ME, Gorio A, Murray M, eds), pp 323-346. Padova, Italy: Liviana.

Rossignol S, Lund JP, Drew T (1988) The role of sensory inputs in regulating patterns of rhythmical movements in higher vertebrates. A comparison between locomotion, respiration and mastication. In: Neural control of rhythmic movements in vertebrates (Cohen AH, Rossignol S, Grillner S, eds), pp 201-283. New York: Wiley.

Rossignol S, Chau C, Brustein E, Bélanger M, Barbeau H, Drew T (1996) Locomotor capacities after complete and partial lesions of the spinal cord. Acta Neurobiol Exp 56:449-463.

Rossignol S, Bélanger M, Chau C, Giroux N, Brustein E, Bouyer L, Grenier T, Drew T, Barbeau H, Reader TA (2000) The spinal cat. In: Neurobiology of spinal cord injury (Kalb RG, Strittmatter SM, eds), pp 57-87. Totowa: Humana.

Roudet C, Giménez y Ribotta M, Privat A, Feuerstein C, Savasta M (1995) Intraspinal noradrenergic-rich implants reverse the increase of alpha 1 adrenoceptors densities caused by complete spinal cord transection or selective chemical denervation: a quantitative autoradiographic study. Brain Res 677:1-12.

Yakovleff A, Roby-Brami A, Guezard B, Mansour H, Bussel B, Privat A (1989) Locomotion in the rats transplanted with noradrenergic neurons. Brain Res Bull 22:115-121.

Yakovleff A, Cabelguen JM, Orsal D, Giménez y Ribotta M, Rajaofetra N, Drian MJ, Bussel B, Privat A (1995) Fictive motor activities in adult chronic spinal rats transplanted with embryonic brainstem neurons. Exp Brain Res 106:69-78. 